\title{
Study on One-Dimensional Wood Board Cutting Stock Problem Based on Adaptive Genetic Algorithm
}

\author{
Wenshu Lin, Dan $\mathrm{Mu}$ and Jinzhuo Wu \\ College of Engineering and Technology, Northeast Forestry University, \\ Harbin 150040, China \\ linwenshu@126.com,*Corresponding author:wujinzhuo1980@163.com
}

\begin{abstract}
When making wood board production, defects on board will influence the machining process automation degree. Therefore, how to fast, accurately remove of wood defects and realize optimal combination cutting stock problem has always been a research hotspot in the field of wood processing. According to the decayed wood board, the paper designed the one dimensional optimization cutting stock combined scheme and mathematical model, adopted the adaptive genetic algorithm imitating the biology evolution to code some optimization scheme initialized by chance, and improved these schemes by selection, crossover and mutation operation. At last these schemes converged to the optimum. The results showed that the adaptive genetic algorithm can achieve a good one dimensional wood board optimization cutting stock problem, through the realization of genetic algorithm in MATLAB, and makes the wood board utilization rate reached $98.9 \%$.
\end{abstract}

Keywords: Adaptive Genetic Algorithm, Optimization Cutting Stock, One-Dimension, Wood Board

\section{Introduction}

Timber is one of the indispensable natural materials in people's daily life. Our country is relatively poor in forest resources. Imported timber accounts for a large portion in timber consumption. The demand for timber will rise more quickly than its supply. In recent years, due to extensive damage and felling to forest resources, forest area is dropping rapidly and it becomes difficult for the supply of timber to meet its demand. Therefore, it is a radical strategy for relieving the increasingly short supply of timber to protect the existing forest resources, make rational use of the limited resources, tap latent resources and improve rate of processing and utilization of timber. Among these measures, to strengthen timber production technology innovation and improve the rate of wood board processing and utilization is a very effective way.

"Cutting stock problem" refers to the division of the same-shape raw materials into a number of parts of different sizes. According to the number of dimensions of the raw material and the parts, the cutting stock problem can be divided into one-, two- and three-dimensional cutting stock problems [1]. The one-dimensional cutting stock problem refers to the case of a number of raw strip materials of a single specification or diversified specifications that are to be cut into various specifications of parts. Aiming at the highest rate of utilization, we need to find out the numbers of each specification of parts. The one-dimensional cutting stock problem is a classic combinatorial optimization problem and it belongs to an NPhard problem [2]. In the past few decades, many scholars at home and abroad made study on the one-dimensional cutting stock problem. The genetic algorithm, 
simulated annealing algorithm and ant colony algorithm are the most popular algorithms used by these researchers [3-7]

In consideration of some weak points of genetic algorithm and insufficient study on optimized cutting of defective wood boards in one-dimensional cutting stock problem, this paper tries to use an adaptive genetic algorithm to determine an optimized wood board cutting plan and to lay a foundation for intelligent automation control for optimized wood board cutting. The study made by this paper can not only reduce labor intensity of workers, but also can improve the rate of production and utilization of timber processing, so as to bring about higher economic benefit to timber processing enterprises in timber production. This is of great importance and impact to the development of intelligent automation of timber processing and to the relief of timber short supply problem in this country.

\section{Genetic Algorithm for Optimized Stock Cutting Combinations}

\subsection{Design of Fitness Function}

Since it is impossible to locate the defects and identify the number of defects in wood boards in wood board processing, the effective wood board length for stock cutting changes with the defects. We need to calculate the effective wood board lengths for each board for stock cutting and then make optimized combination. The problem of optimized wood board cutting combination is a study on how to choose the optimized nesting arrangement among a variety of nesting plans.

Suppose we have a wood board, with effective length L for cutting. The board will be cut into $m$ kinds of small board parts with length $l_{j}$, (where $j=1,2,3 \ldots \mathrm{m}$ and $j$ denotes the $j$-th kind board part; $m \geq 1$ and $\left.l_{j} \leq L\right)$. $a_{j}$ is the quantity of each kind of possible small board parts. The length of residual wood board from a certain cutting plan is $b_{i}$ (i represents the $\mathrm{i}$-th cutting plan in the cutting plans, $\mathrm{i}=1,2,3 \ldots$ n). The cutting plan can be expressed by the expression as follows:

$b_{i}=L-\left(a_{i 1} \times l_{1}+a_{i 2} \times l_{2}+\cdots+a_{i m} \times l_{m}\right) \quad\left(b_{i} \geq 0\right)$

1)

If a term in the expression happens to be 0 , it means the board part of such size shall not be cut in this cutting plan. In the expression, the wood board length and the board part lengths are known. The lengths of board parts $l_{1}, l_{2}, \ldots \ldots l_{m}$ are arranged in descending order. To find the i-th cutting plan means to find $a_{i m}$. The set of coefficients represent the quantity of each size of small board parts. Substituting the set of coefficients in the expression, we can obtain the value of residual material $b_{i}$, if the wood board is cut by this cutting method. Compare is made to find the optimized plan of all the cutting plans with the minimum $b_{i}$. Formula (1) is the objective function for optimized combination for wood board cutting. Therefore, the formula is taken as the fitness function in genetic algorithm.

\subsection{Individual Encoding and Generation of Initial Population}

In this paper, binary encoding is adopted. For an individual, coefficient $a_{i m}$ is used to express the chromosome code generated from binary encoding. The coefficients shall then be yhlined up in a certain sequence. The scope of value for coefficient $a_{i m}$ : the lower limit is 0 and the upper limit is determined by the code when coefficient $a_{i m}$ reaches its maximal value. Each coefficient takes a random value within the scope of value, followed 
by binary encoding. After chromosomes are determined, all chromosomes shall be sorted in order based on the size of board parts required. Then, an individual is formed. Each individual represents one optimization plan.

Suppose we have a wood board with a length $\mathrm{L}$ of $321 \mathrm{~cm}$. The board will be cut into three sizes of small board parts: $l_{1}=54 \mathrm{~cm}, l_{2}=46 \mathrm{~cm}, l_{3}=31 \mathrm{~cm}$. The coefficient $a_{1}$ of the required board part $l_{1}$ shall take a value with the scope of [0, 5]. The string length of chromosome $S_{1}$ is 3 digits. The coefficient $a_{2}$ shall take a value with the scope of $[0,6]$. The string length of chromosome $S_{2}$ is 3 digits. The coefficient $a_{3}$ shall take a value with the scope of $[0,10]$. The string length of chromosome $S_{3}$ is 4 digits. So, the total string length of chromosomes of individual codes in the initial population is 10 digits. The form of expression is shown in Figure 1.

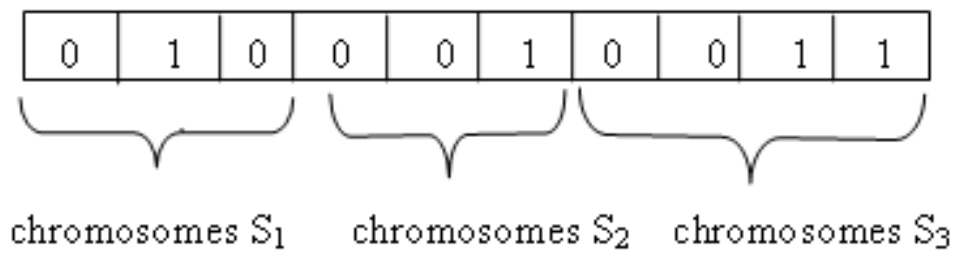

Figure 1. Chromosomes of Individual Form

The convergence rate will be affected by initial population size during genetic process. In order to achieve better convergence results, the size of the initial population must be chosen properly. If it is chosen improperly, the result of genetic algorithm can be seriously affected. A large population size will cause too-fast convergence and lead to unsatisfactory results. The quantity of required board parts is low in this paper, so the population size is very limited. The method to generate initial population is a uniform distribution over the space of solutions. We assume the initial population consists of 20 individuals.

\subsection{Selection, Crossover and Mutation Operations}

Selection operation is the basis for continuous genetic operation of the population. In accordance with the mechanisms of biological evolution and natural selection theory, an individual with good nature will have higher probability to be selected. Crossover and mutation operations will lead to the population of the next generation. In this paper, roulette wheel selection method is used. This is a relatively simple selection method. The probability of individual fitness is converted into a probability of being selected. The individual with higher fitness will be selected with a higher probability.

The purpose of crossover operation is to be able to generate new individuals in the next generation just like human marriage. The search capacity of genetic algorithm is significantly improved through crossover operation. Crossover is the most important process to obtain new superior individuals in genetic algorithm. In consideration of the length of individuals, single-point crossover is adopted. The crossover points are randomly generated.

Through offspring mutation after crossover, the offspring individuals will change according to mutation probability $P_{m}$. The number of variables changes in proportion to the individual variable probability, but has nothing to do with the size of population. If this digit is 0 , the mutation is 1 . Otherwise, it will be 0 . After generation of a new population through selection, crossover and mutation, judgment shall be made on population individuals based on optimization criteria. If 
optimization criteria are met, the process shall terminate. Otherwise, the process continues.

\subsection{Termination Condition}

There are two types of common termination conditions for genetic algorithms. One is to set the maximum (genetic) number of generations. The other is to make judgment based on individual difference to take control through calculation of genetic diversity measurement or the similarity of all genes in a population. The first method is adopted by this paper and the maximum evolution generation is set to be 50 .

\section{Treatment of Defects of Wood Board}

The paper focuses on cutting of wood board of a single effective length. But in actual production, more than one defect may exist on a piece of wood board, so it can be divided into a number of effective wood boards that can be cut. Then optimal combination is made to each of such effective wood boards that can be cut. The cutting position is determined according to the position of defects and optimization plan (as shown in Figure 2). The first section from the right side is positioned relative to the right end surface. The middle part is positioned relative to the defect on its right. The last section of the wood board is positioned relative to the left end. In this way, we can save wood board cutting rounds, save timber and improve timber productivity.

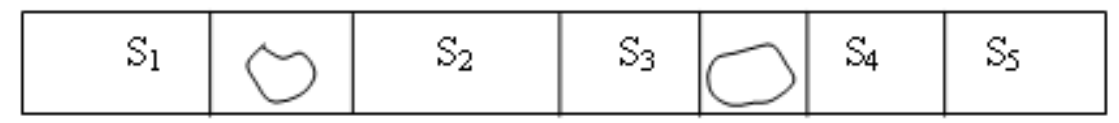

Figure 2. Defect Board Sawing Position

\section{Results of Operation}

In the genetic algorithm in this study, the population size is set to 20 , the number of breeding generations being 20, the crossover probability being 0.9 and the mutation probability being 0.1 . According to the assumed wood board length for cutting into three sizes of small board parts and the coefficients for various sizes described in paragraph 2.2, running the prepared genetic algorithm in software MATLAB, the results of operation are shown in Figure 3 and Figure 4.

In Figure 3, the abscissa represents the number of the population, and the vertical axis represents the residual material. $x$ represents the number of board parts of $54 \mathrm{~cm}$ long. $y$ represents the number of board parts of $46 \mathrm{~cm}$ long, and $\mathrm{z}$ represents the number of board parts of $31 \mathrm{~cm}$ long, and $\mathrm{x}=3$ means there are three board parts of such size. $\mathrm{y}=0$ means no cutting for board parts of $46 \mathrm{~cm}$ long. $\mathrm{z}=5$ means there are 5 board parts of the corresponding size. In addition, we can see from Fig. 3 that optimization is carried forward from a generation to the next generation by adaptive genetic algorithm with convergence to the optimal solutions through 20 rounds of iterations at last. Based on the initial wood board length of $321 \mathrm{~cm}$, the residual material is $4 \mathrm{~cm}$ long. The rate of utilization of the wood board is as high as $98.8 \%$. Therefore, the adaptive genetic algorithm designed in this paper can effectively and quickly solve one-dimensional defective wood board cutting optimization problem. 


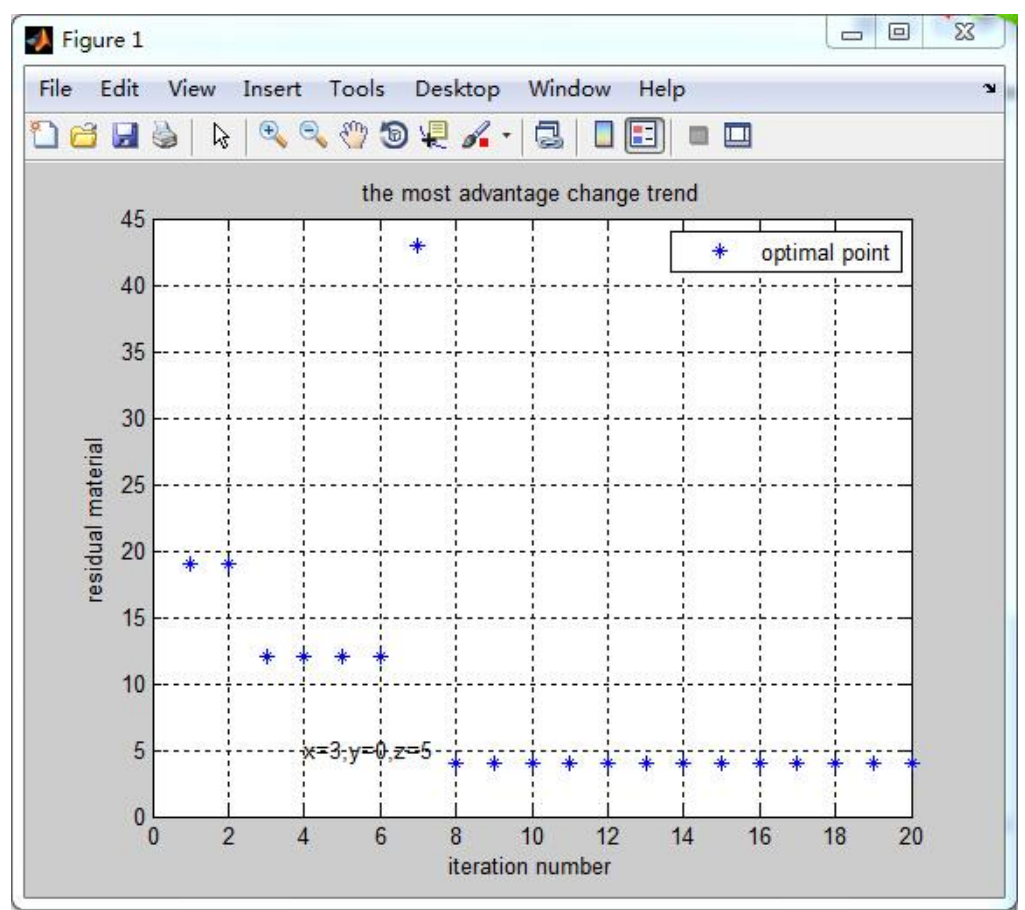

Figure 3. The Trend of the Most Advantage Point

At the same time, we can get the optimal binary codes of chromosomes, as shown in Figure 4, where the abscissa indicates the length of chromosomes and the vertical axis represents the value of the binary numbers. Therefore, the optimized individual is represented by the binary number of 1010011,000.

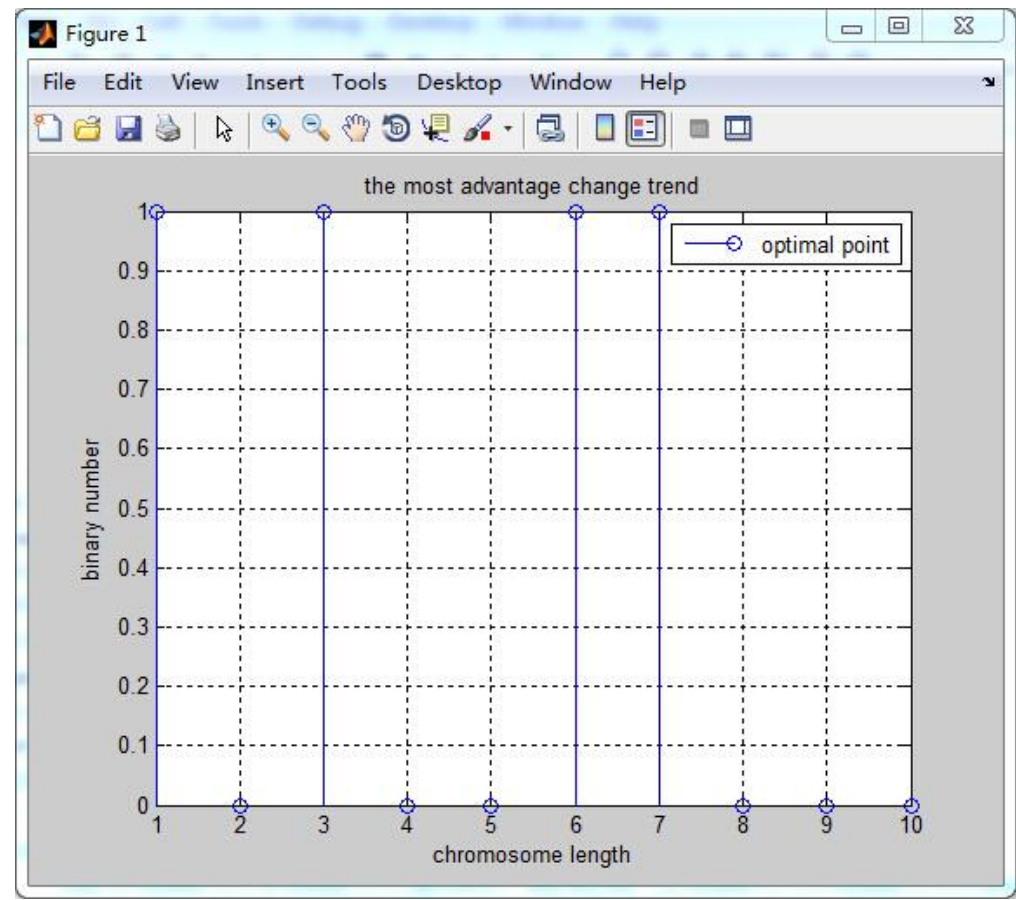

Figure 4. Optimal Binary Chromosome Representation

\section{Conclusions}


Genetic algorithm, as an artificial intelligence technology, is used in this paper to perform optimization combination for a solution to one-dimensional wood board cutting plan. Adaptive genetic algorithm is used to perform optimization calculation of the defectrelated information of wood board and set up a mathematical model for optimized onedimensional wood board cutting problem. With minimum residual material as the objective function, genetic algorithm runs in MATLAB. The optimal one-dimensional wood board cutting plan is finally determined through optimization treatment by selection, crossover and mutation. The result of this study can improve rate of wood board utilization and productivity, so as to avoid waste of large quantity of timber. Meanwhile, the adaptive genetic algorithm designed in this paper can find application in the stock cutting problems of other types of sheet materials.

\section{Acknowledgements}

The authors would like to acknowledge the support of the Fundamental Research Funds for the Central Universities (DL13BB19) and the Scientific Activities Support Program of Chinese State Forestry Administration for Returned Talents from Overseas (415003).

\section{References}

[1] C.L. Zhang and Y.D. Cui, "Optimization of one dimensional cutting stock problem. Journal of Guilin Institute of Technology", vol. 24, no. 1, (2001), pp. 103-106.

[2] W.S. Xing and J.X. Xie, "Editor, Modern Optimization Method", Tsinghua University Press, Beijing, (1999).

[3] S. P. Jin, D. F. Chen, X. Zhang and S. L. Dai, "A genetic algorithm for cutting one dimension material", Journal of Wuhan Transportation University, vol. 21, no. 2, (1997), pp. 168-172.

[4] D. Wu, C. R. Li and G. J. Song, "Bee swarm genetic algorithm for one-dimensional cutting problem", Computer Technology and Development", vol. 20, no. 10, (2010), pp. 82-85.

[5] Z. J. Wu, L. P. Zhang and K. Wang, "An ant colony algorithm for one dimensional cutting stock problem", Mechanical Science and Technology for Aerospace Engineering, vol. 27, no. 12, (2008), pp. 1681-1684.

[6] Z. X. Jia, F. Q. Yin, X. B. Hu and B. Shu, "Optimization for one-dimensional cutting-stock problem based on genetic", Journal of Xi'an Jiaotong University, vol. 36, no. 9, (2002), pp. 967-970.

[7] G. J. Zhang and X. J. Xu, "Application of genetic algorithm to optimization of one-dimensional cuttingstock without replicated sizes", Computer Simulation, vol. 25, no, (2008), 3, pp. 275-277.

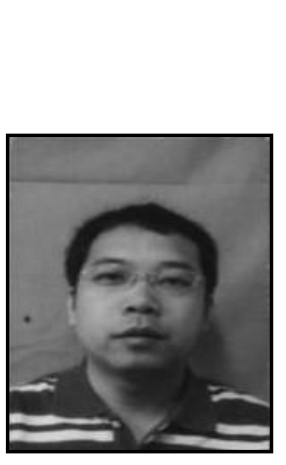

\section{Authors}

Wenshu Lin earned a B.E. degree and a M.S. degree in Forest Engineering from Northeast Forestry University, Harbin, P. R. China in 2003 and 2005, respectively. In 2011, he received a Ph.D. degree in Forest Resources Science from West Virginia University, USA. He is a Member of Forest Products Society and Society of Wood Science and Technology. Dr. Lin had several publications in esteemed journals such as Forest Products Journal, Wood and Fiber Science, and Computers and Electronics in Agriculture. Currently, he is an associate professor at Northeast Forestry University, Harbin, P. R. China. His major research interests include nondestructive testing and evaluation of wood, wood utilization and production improvement, and computer simulation and system modeling. 

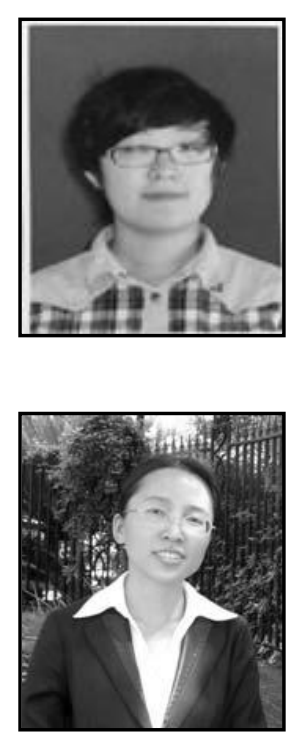

Dan Mu earned a B.E. degree in Forest Engineering from Northeast Forestry University, Harbin, P.R. China in 2014. Currently, she is a master student in the Northeast Forestry University. She major research interests include nondestructive testing and evaluation of wood, cutting stock problem, and computer simulation and system modeling.

Jinzhuo Wu earned her B.E. degree and M.S. degree in Forest Engineering from Northeast Forestry University, Harbin, P.R. China in 2003 and 2005, respectively. In 2010, she obtained her Ph.D. degree in Forest Resources Science from West Virginia University, USA. She is a Member of Forest Products Society. Dr. Wu had several publications in esteemed journals such as Forest Products Journal, Wood and Fiber Science, Canadian Journal of Forest Research, and Journal of Agriculture and Resources Economics. 
International Journal of Future Generation Communication and Networking Online Vol. 9, No. 4, (2016) 